\title{
MAGNETIC MOMENTS OF CHARM BARYONS IN CHIRAL PERTURBATION THEORY
}

\author{
V.S. Zamiralov ${ }^{1}$ \\ D.V. Skobeltsyn Institute of Nuclear Physics, Moscow State University, \\ Moscow, Russian Federation \\ and \\ The Abdus Salam International Centre for Theoretical Physics, Trieste, Italy.
}

\begin{abstract}
Magnetic moments of the charm baryons of the sextet and of the $3^{*}$-plet are re-evaluated in the framework of Heavy Hadron Chiral Perturbation Theory (HHCPT). NRQM and broken $S U(4)$ unitary symmetry model are used to obtain tree-level magnetic moments. Calculations of a unitary symmetry part of one-loop contributions to magnetic moments of the charm baryons are performed in detail in terms of the $S U(4)$ couplings of charm baryons to Goldstone bosons. The relations between the magnetic moments of the sextet $1 / 2$ baryons with the one-loop corrections are shown to coincide with the NRQM relations. The correspondence between HHCPT results and those of NRQM and unitary symmetry model is discussed. It is shown that one-loop corrections can effectively be absorbed into the tree-level formulae for the magnetic moments of the charm baryons in the broken $S U(4)$ unitary symmetry model and partially in the NRQM.
\end{abstract}

\section{MIRAMARE - TRIESTE}

November 2001

\footnotetext{
${ }^{1}$ E-mail: zamir@depni.npi.msu.su
} 


\section{Introduction}

Magnetic moments of charm baryons were first described in the quark model with four flavors [1] and $S U(4)_{f}$ unitary symmetry model and its extension to $S U(8) \supset U(4)_{f} \times S U(2)$ [2]. Later, when a specific role of heavy quark inside heavy hadron were realized $[3,4,5]$ in an approach based on the heavy quark effective theory and chiral perturbation theory, leading long-distance contribution to magnetic moments of the charm antitriplet were computed in [6]. Recently in a similar approach magnetic moments of heavy baryons were analyzed [7]. One-loop corrections were calculated and relations are obtained between magnetic moments of sextet charm and beauty baryons [7]. These relations, as we shall see, are those of a simple quark model [1] even in the presence of one-loop corrections of order $\mathcal{O}\left(1 / \Lambda_{\chi}^{2}\right)$ where $\Lambda_{\chi}$ is a chiral symmetry breaking scale. As this is not the case for the magnetic moments of octet baryons and in order to make a more direct comparison of magnetic moments of the charm baryons with those of the octet baryons I re-evaluate here magnetic moments of the charm baryons following the approach of $[8,9,10]$ for the octet and decuplet baryons and taking into account one-loop corrections in the framework of the HHCPT in terms of $S U(4)$ coupling constants for charm baryons and Goldstone bosons.

Magnetic moments at the tree level can be taken either from a quark model (see, e.g., [1]) or from a unitary symmetry model (see, e.g., [2]). These models could be put in a form which corresponds effectively to a description of the tree-level magnetic moments in the framework of the HHCPT as the sum of the terms of order $\mathcal{O}\left(1 / \Lambda_{\chi}\right)$ arising due to light quark contributions, and the terms of order $\mathcal{O}\left(1 / m_{c}\right)$ arising due to a charm quark contribution.

All masses of the charm $1 / 2$ and $3 / 2$ baryons are taken to be degenerated.

The plan of the paper is the following. First I write the necessary elements of the HHCPT. Then tree-level contributions are written in the frameworks of the NRQM and $S U(4)_{f}$ unitary symmetry model. In the 4th section one-loop contributions into the charm baryon magnetic moments are evaluated one by one and the corresponding Feynman diagrams are presented. Results are compared with those of [7]. In the 5th section relations between the charm baryon magnetic moments are given and discussed.

\section{Elements of the HHCPT Formalism}

I write the necessary elements of the Heavy Hadron Chiral Perturbation Theory (HHCPT), basing mainly on the works of $[7,8,9,10]$. In its framework a chiral expansion of the heavy (charm in our case) baryon Lagrangian is written in terms of the velocity-dependent fields $B_{v}(x)$ constructed in order to remove free momentum dependence in Dirac equation,

$$
B_{v}(x)=\exp \left(i M_{B} \hat{v} v \cdot x\right) B(x)
$$


Here $B(x)$ is a charm baryon $1 / 2$ field with central mass $M_{B}$. It would be more convenient for us to work not with the 6 - and $3^{*}$-plets of the charm baryons as in [7] but instead with the whole $S U(4)_{f} 20_{4}^{\prime}$-plet. The $20_{4}^{\prime}$-plet $B_{v}(x)$ is given by the 3 rd-rang tensor $B_{\beta \gamma}^{\alpha} \quad \alpha, \beta, \gamma=$ $1,2,3,4\left(B_{\beta \gamma}^{\alpha}=-B_{\gamma \beta}^{\alpha}, B_{\alpha \beta}^{\alpha}=0\right)$. Usually one can make a reduction of the baryon $20_{4}^{\prime}$-plet to the multiplets of the $S U(3)_{f}$-group with the definite value of the charm quantum number as $20_{4}^{\prime}=8_{3}+6_{3}+3_{3}^{*}+3_{3}$. The ordinary octet $8_{3}$ with $C=0$ is:

$$
B_{v}=\left(\begin{array}{ccc}
\frac{1}{\sqrt{2}} \Sigma_{v}^{0}+\frac{1}{\sqrt{6}} \Lambda_{v}^{0} & \Sigma_{v}^{+} & p_{v} \\
\Sigma_{v}^{-} & -\frac{1}{\sqrt{2}} \Sigma_{v}^{0}+\frac{1}{\sqrt{6}} \Lambda_{v}^{0} & n_{v} \\
\Xi_{v}^{-} & \Xi_{v}^{0} & -\frac{2}{\sqrt{6}} \Lambda_{v}^{0}
\end{array}\right) .
$$

Charm baryons of the sextet $6_{3}$ and antitriplet $3_{3}^{*}$ with $C=1$ are given by the tensors $B_{b c}^{a}$ and $B_{b 4}^{4}, a, b, c=1,2,3^{2}$. Note that in the quark model the $6{ }_{3}$-plet charm baryons are all $\Sigma$-like ones with two light quarks in a symmetrical state and the charm quark apart:

$$
\begin{gathered}
\Sigma_{c}^{++}(u u, c)=B_{23}^{1}, \quad \Sigma_{c}^{0}(d d, c)=B_{13}^{2}, \quad \Omega_{c}^{0}(s s, c)=B_{12}^{3}, \\
\sqrt{2} \Sigma_{c}^{+}(u d, c)=B_{13}^{1}-B_{23}^{2}, \quad \sqrt{2} \Xi_{c}^{\prime+}(u s, c)=B_{12}^{1}-B_{32}^{3}, \\
\sqrt{2} \Xi_{c}^{\prime 0}(d s, c)=B_{12}^{2}-B_{13}^{3} .
\end{gathered}
$$

Instead $3_{3}^{*}$-plet charm baryons are all $\Lambda$-like ones:

$$
B_{14}^{4}=\frac{2}{\sqrt{6}} \Xi_{c}^{0}(c s d), \quad B_{24}^{4}=\frac{2}{\sqrt{6}} \Xi_{c}^{+}(c s u), \quad B_{34}^{4}=\frac{2}{\sqrt{6}} \Lambda_{c}^{+}(c u d) .
$$

The $3 / 26_{3}$-plet charm baryons $T_{\mu}^{a b 4}, a, b=1,2,3$, are a part of the $S U(4)_{f} 20$-plet $\left(20_{4}=\right.$ $10_{3}+6_{3}+3_{3}+1_{3}$. ) and in the quark model all have quarks in a symmetrical state:

$$
\begin{array}{cc}
\Sigma_{c}^{*++}(u u c)=T^{114}, \sqrt{2} \Sigma_{c}^{*+}(u d c)=T^{124}, & \Sigma_{c}^{* 0}(d d c)=T^{224}, \\
\sqrt{2} \Xi_{c}^{*+}(u s c)=T^{134}, \quad \sqrt{2} \Xi_{c}^{* 0}(d s c)=T_{234}^{2}, & \Omega_{c}^{* 0}(s s c)=T^{334} .
\end{array}
$$

Goldstone bosons appearing in the limit of chiral symmetry are identified with the pseudoscalar octet and are parametrized as follows

$$
P=\frac{1}{\sqrt{2}}\left(\begin{array}{ccc}
\frac{1}{\sqrt{2}} \pi^{0}+\frac{1}{\sqrt{6}} \eta & \pi^{+} & K^{+} \\
\pi^{-} & -\frac{1}{\sqrt{2}} \pi^{0}+\frac{1}{\sqrt{6}} \eta & K^{0} \\
K^{-} & \bar{K}^{0} & -\frac{2}{\sqrt{6}} \eta
\end{array}\right) .
$$

This pseudoscalar octet couples to the baryon fields via the vector and axial vector currents

$$
V^{\mu}=\frac{1}{2}\left(\xi \partial^{\mu} \xi^{\dagger}+\xi^{\dagger} \partial^{\mu} \xi\right), \quad A^{\mu}=\frac{i}{2}\left(\xi \partial^{\mu} \xi^{\dagger}-\xi^{\dagger} \partial^{\mu} \xi\right)
$$

where $\xi=\exp (i P / f)$ and $\xi \rightarrow L \xi R^{\dagger}$, with $L, R \in S U(3)_{L, R}$ and $f=f_{\pi} \approx 93 M e V$ being the pseudoscalar decay constant in the chiral limit.

\footnotetext{
${ }^{2}$ While using tensor notation here I omit the subscript $v$.
} 
The lowest order Lagrangian (in notations of $[8,9,10]$ ) reads

$$
L=L^{1 / 2}+L^{3 / 2 \rightarrow 1 / 2}+L^{3 / 2}+\frac{f_{\pi}^{2}}{4} \operatorname{Tr}\left(\partial^{\mu} \Sigma^{\dagger} \partial_{\mu} \Sigma\right)
$$

with $\Sigma=\exp (2 i P / f) \equiv \xi^{2}$ and $L^{1 / 2}, \quad L^{3 / 2 \rightarrow 1 / 2}, \quad L^{3 / 2}$ written below. As it was said it would be convenient for us to write formally vector and axial-vector baryon currents and their couplings to the Goldstone bosons in terms of the $S U(4)$ unitary symmetry model and only then to reduce formulae to the case of the $S U(3)$ Goldstone bosons, given by the familiar octet of the pseudoscalar mesons $\pi, K$ and $\eta$. It would also be convenient in what follows to write the corresponding Lagrangian of the electromagnetic interaction of the baryons through the $S U(4)$ electromagnetic baryon currents and then to extract charm baryon piece.

As the pseudoscalar octet is a part of the $S U(4)_{f} 15_{4}$-plet $\left(15_{4}=8_{3}+3_{3}^{*}+3_{3}+1_{3}\right)$ I write formally the interaction of the latter with the baryons of the $20_{4}^{\prime}$-plet through a $S U(4)$ invariant Lagrangian

$$
\begin{array}{r}
L^{1 / 2}=i \operatorname{Tr}\left(\bar{B}_{v} v \cdot \mathcal{D} B_{v}\right)+2(-F+D)\left(\bar{B}_{v \gamma}^{\alpha \beta} S_{v}^{\mu} A_{\mu \alpha}^{\rho} B_{v \rho \beta}^{\gamma}\right)+ \\
2(F+D)\left(\bar{B}_{v \rho}^{\alpha \beta} S_{v}^{\mu} B_{v \alpha \beta}^{\gamma}\right) A_{\mu \gamma}^{\rho}, \quad \mathcal{D}_{\mu} B_{v}=\partial_{\mu} B_{v}+\left[V_{\mu}, B_{v}\right],
\end{array}
$$

and then take the part corresponding to the interaction of the charm baryons with the light Goldstone bosons $P_{b}^{a}, a, b=1,2,3$. In the same way transition current between the charm 6 -plet $3 / 2$ and the charm 6 -plet $1 / 2$ can be written first in terms of the $20_{4}^{\prime}$-plet $1 / 2$ and the $20_{4}$-plet $3 / 2\left(20_{4}=10_{3}+6_{3}+3_{3}+1_{3}\right)$ which interacts with the $15_{4}$-plet Goldstone bosons through a Lagrangian:

$$
L^{3 / 2 \rightarrow 1 / 2}=\mathcal{C}\left(\epsilon_{\beta \gamma \rho a} \bar{B}_{v \alpha}^{\beta \gamma} T_{v}^{\mu \alpha \rho \tau} A_{\mu \beta}^{\delta}+\epsilon^{\beta \gamma \rho \delta} \bar{T}_{v \mu \alpha \rho \beta} B_{v \beta \gamma}^{\alpha} A_{\delta}^{\mu \beta}\right)
$$

Then we take the part corresponding to the interaction of the charm 6-plets with the light Goldstone bosons $P_{b}^{a}, a, b=1,2,3$. Similarly I also write $S U(4)$ invariant Lagrangian for the interaction of the $20_{4}$-plet formally with the $15_{4}$-plet Goldstone bosons:

$$
\begin{array}{r}
L^{3 / 2}=\bar{T}_{v \alpha \rho \eta}^{\mu} v \cdot \mathcal{D} T_{v \mu}^{\alpha \rho \delta}+2 \mathcal{H} \bar{T}_{v \alpha \rho \eta}^{\mu} S_{v \nu} A_{\beta}^{\nu} \delta T_{v \mu}^{\alpha \rho \beta}, \\
\mathcal{D}^{\nu} T_{v \mu}^{\alpha \rho \delta}=\partial^{\mu} T_{v \mu}^{\alpha \rho \delta}+\left(V^{\nu}\right)_{\beta}^{\alpha} T_{v \mu}^{\beta \rho \delta}+\left(V^{\nu}\right)_{\beta}^{\rho} T_{v \mu}^{\alpha \beta \delta}+\left(V^{\nu}\right)_{\beta}^{\delta} T_{v \mu}^{\alpha \rho \beta},
\end{array}
$$

and then take the part corresponding to the interaction of the charm 6 -plet $3 / 2$ from $20_{4}$-plet with the light Goldstone bosons $P_{b}^{a}, a, b=1,2,3$.

Extracting from Eqs. $(3,4,5)$ the $S U(3)$ symmetry part with octet baryons $(1)$, one arrives at the effective Lagrangians used in [9] for the one-loop correction calculations for the octet and decuplet magnetic moments with the same constants $F, D, \mathcal{C}, \mathcal{H}$. 


\section{Tree-level magnetic moments}

\section{Nonrelativistic quark model}

Let us now briefly discuss tree-level magnetic moments beginning from the quark model. In the 4-flavor nonrelativistic quark model all the moments can be expressed in terms of the 4 quark magnetons $\mu_{q}, \quad q=u, d, s, c$. The 6-plet charm baryon magnetic moments are [1]:

$$
\begin{array}{r}
\mu_{\Sigma_{c}^{++}}=\frac{4}{3} \mu_{u}-\frac{1}{3} \mu_{c}, \quad \mu_{\Sigma_{c}^{+}}=\frac{2}{3} \mu_{u}+\frac{2}{3} \mu_{d}-\frac{1}{3} \mu_{c} \\
\mu_{\Sigma_{c}^{0}}=\frac{4}{3} \mu_{d}-\frac{1}{3} \mu_{c}, \quad \mu_{\Omega_{c}^{0}}=\frac{4}{3} \mu_{s}-\frac{1}{3} \mu_{c}, \\
\mu_{\Xi_{c}^{\prime+}}=\frac{2}{3} \mu_{u}+\frac{2}{3} \mu_{s}-\frac{1}{3} \mu_{c}, \quad \mu_{\Xi_{c}^{\prime 0}}=\frac{2}{3} \mu_{d}+\frac{2}{3} \mu_{s}-\frac{1}{3} \mu_{c} .
\end{array}
$$

In the HHPCT a natural scale of the leading order contributions to the magnetic moments would be $\mathcal{O}\left(1 / \Lambda_{\chi}\right)$ for $\mu_{u, d, s}$ and $\mathcal{O}\left(1 / m_{c}\right)$ for $\mu_{c}[7,10]$. Formally with $\mu_{u}=-2 \mu_{d}=-2 \mu_{s}=c_{s} / 3 \Lambda_{\chi}$ and $\mu_{c}=-1 / 6 m_{c}$ we arrive at the first two terms of Eq.(15) in [7] in their notations and their Table 1 taken into account.

The following relations are valid in NRQM:

$$
\begin{array}{r}
\mu_{\Sigma_{c}^{++}}+\mu_{\Sigma_{c}^{0}}=2 \mu_{\Sigma_{c}^{+}}, \\
\mu_{\Sigma_{c}^{++}}+\mu_{\Omega_{c}^{0}}=2 \mu_{\Xi_{c}^{\prime+}}, \\
\mu_{\Sigma_{c}^{++}}+2 \mu_{\Xi_{c}^{\prime 0}}=\mu_{\Sigma_{c}^{0}}+2 \mu_{\Xi_{c}^{\prime+}} .
\end{array}
$$

We shall see later that the same relations are valid also with the one-loop corrections included.

The $3^{*}$-plet charm baryon magnetic moments in NRQM are all equal [1]:

$$
\mu_{\Lambda_{c}^{+}}=\mu_{\Xi_{c}^{+}}=\mu_{\Xi_{c}^{0}}=\mu_{c}
$$

In the HHPCT a natural scale for leading order contribution would be $\mathcal{O}\left(1 / m_{c}\right)$. Formally the quark model result can be related to that of HHPCT in [7]. It coincides with the first term of Eq.(33) in [7] with $\mu_{c}=-1 / 6 m_{c}$. It is valid up to the order $\mathcal{O}\left(1 / \Lambda_{\chi}\right)$ as non-zero corrections begin only at the order $\mathcal{O}\left(1 / m_{c} \Lambda_{\chi}\right)[7]$.

\section{Unitary symmetry model}

Magnetic moments of the charm baryons at the tree level can be evaluated in the framework of the $S U(4)_{f}$ unitary symmetry model [2]. Electromagnetic baryon current transforms under the reducible group representation $15+1$, and we choose to introduce symmetry breaking terms due to strangeness and charm. Then magnetic moments would be given by the current :

$$
\begin{array}{r}
J_{\mu}^{e l-m a g}=\left(g_{1} \bar{B}_{\gamma}^{1 \beta} \gamma_{\mu} B_{1 \beta}^{\gamma}+g_{1}^{\prime} \bar{B}_{\gamma}^{4 \beta} \gamma_{\mu} B_{4 \beta}^{\gamma}+h_{1} \bar{B}_{\gamma}^{3 \beta} \gamma_{\mu} B_{3 \beta}^{\gamma}\right)+ \\
\frac{1}{2}\left(g_{2} \bar{B}_{1}^{\alpha \beta} \gamma_{\mu} B_{\alpha \beta}^{1}+g_{2}^{\prime} \bar{B}_{4}^{\alpha \beta} \gamma_{\mu} B_{\alpha \beta}^{4}+h_{2} \bar{B}_{3}^{\alpha \beta} \gamma_{\mu} B_{\alpha \beta}^{3}\right)- \\
\frac{1}{3}\left(g_{1}+g_{2}+g_{1}^{\prime}-2 g_{0}-3 h_{1}\right) S p\left(\bar{B} \gamma_{\mu} B\right)
\end{array}
$$


With $g_{1}=g_{1}^{\prime}=-\frac{1}{2} \mu_{x}, g_{2}=g_{2}^{\prime}=-\frac{1}{2} \mu_{y}, h_{1}=h_{2}=0$ one returns to the $S U(4)_{f}$-symmetric electromagnetic current given in [2]. With $g_{1}=-\mu_{F}+\mu_{D}, g_{2}=\mu_{F}+\mu_{D}, h_{1}=-\mu_{F}^{s}+\mu_{D}^{s}$, $h_{2}=\mu_{F}^{s}+\mu_{D}^{s}, g_{0}=-g_{1}^{\prime}$ one arrives at the $S U(3)_{f}$ formulae for the octet baryon magnetic moments with corrections due to strangeness in terms of the constants $\mu_{F}, \mu_{D}, \mu_{F}^{s}, \mu_{D}^{s}$ (similar to those written in [12]):

$$
\begin{array}{r}
\mu_{p}=\mu_{F}+\frac{1}{3} \mu_{D}, \quad \mu_{n}=-\frac{2}{3} \mu_{D}, \\
\mu_{\Sigma^{+}}=\frac{4}{3} \mu_{F}+\frac{1}{3}\left(\mu_{D}^{s}-\mu_{F}^{s}\right), \quad \mu_{\Sigma^{-}}=-\frac{2}{3} \mu_{F}+\frac{1}{3}\left(\mu_{D}^{s}-\mu_{F}^{s}\right), \\
\mu_{\Xi^{0}}=\frac{4}{3} \mu_{F}^{s}+\frac{1}{3}\left(\mu_{D}-\mu_{F}\right), \quad \mu_{\Xi^{-}}=-\frac{2}{3} \mu_{F}^{s}+\frac{1}{3}\left(\mu_{D}-\mu_{F}\right), \\
\mu_{\Lambda}=\frac{1}{3}\left(\mu_{F}-\frac{2}{3} \mu_{D}\right)-\frac{1}{3}\left(\mu_{F}^{s}+\frac{1}{3} \mu_{D}^{s}\right) .
\end{array}
$$

Disregarding superscript 's ', one obtains known $S U(3)_{f}$ results [11]. The 6-plet magnetic moments are given by $\left(\rightarrow\right.$ means $S U(4)_{f}$ limit):

$$
\begin{aligned}
& \mu_{\Sigma_{c}^{++}}=\frac{4}{3} \mu_{F}+\frac{2}{3}\left(\mu_{F}^{c}-\mu_{D}^{c}\right) \rightarrow 2 \mu_{F}-\frac{2}{3} \mu_{D}, \\
& \mu_{\Sigma_{c}^{+}}=\frac{1}{3} \mu_{F}+\frac{2}{3}\left(\mu_{F}^{c}-\mu_{D}^{c}\right) \rightarrow \mu_{F}-\frac{2}{3} \mu_{D}, \\
& \mu_{\Sigma_{c}^{0}}=-\frac{2}{3} \mu_{F}+\frac{2}{3}\left(\mu_{F}^{c}-\mu_{D}^{c}\right) \rightarrow-\frac{2}{3} \mu_{D}, \\
& \mu_{\Xi_{c}^{\prime+}}=\frac{2}{3} \mu_{F}-\frac{1}{3} \mu_{F}^{s}+\frac{2}{3}\left(\mu_{F}^{c}-\mu_{D}^{c}\right) \rightarrow \mu_{F}-\frac{2}{3} \mu_{D}, \\
& \mu_{\Xi_{c}^{\prime 0}}=-\frac{1}{3} \mu_{F}-\frac{1}{3} \mu_{F}^{s}+\frac{2}{3}\left(\mu_{F}^{c}-\mu_{D}^{c}\right) \rightarrow-\frac{2}{3} \mu_{D}, \\
& \mu_{\Omega_{c}^{0}}=-\frac{2}{3} \mu_{F}^{s}+\frac{2}{3}\left(\mu_{F}^{c}-\mu_{D}^{c}\right) \rightarrow-\frac{2}{3} \mu_{D},
\end{aligned}
$$

with

$$
\begin{gathered}
g_{1}^{\prime}=\frac{1}{3} g_{1}+\frac{2}{3} g_{1}^{c}, \quad g_{2}^{\prime}=\frac{1}{3} g_{2}+\frac{2}{3} g_{2}^{c} \\
g_{1}^{c}=-\mu_{F}^{c}+\mu_{D}^{c}, \quad g_{2}^{c}=\mu_{F}^{c}+\mu_{D}^{c}
\end{gathered}
$$

The $3^{*}$-plet magnetic moments are given instead by :

$$
\begin{array}{r}
\mu_{\Xi_{c}^{0}}=-\frac{1}{3}\left(\mu_{F}-\frac{2}{3} \mu_{D}\right)-\frac{1}{3}\left(\mu_{F}^{s}-\frac{2}{3} \mu_{D}^{s}\right)+\frac{2}{3}\left(\mu_{F}^{c}+\frac{1}{3} \mu_{D}^{c}\right) \rightarrow \frac{2}{3} \mu_{D}, \\
\mu_{\Xi_{c}^{+}}=\frac{2}{3}\left(\mu_{F}-\frac{2}{3} \mu_{D}\right)-\frac{1}{3}\left(\mu_{F}^{s}-\frac{2}{3} \mu_{D}^{s}\right)+\frac{2}{3}\left(\mu_{F}^{c}+\frac{1}{3} \mu_{D}^{c}\right) \rightarrow \mu_{F}, \\
\mu_{\Lambda_{c}^{+}}=\frac{1}{3}\left(\mu_{F}-\frac{2}{3} \mu_{D}\right)+\frac{2}{3}\left(\mu_{F}^{c}+\frac{1}{3} \mu_{D}^{c}\right) \rightarrow \mu_{F}
\end{array}
$$

In the HHPT a natural scale of the leading order contributions to the magnetic moments would be $\mathcal{O}\left(1 / \Lambda_{\chi}\right)$ for $\mu_{F}, \mu_{D}, \mu_{F}^{s}, \mu_{D}^{s}$ and $\mathcal{O}\left(1 / m_{c}\right)$ for $\mu_{F}^{c}, \mu_{D}^{c}$. Formally with $\mu_{F}=\mu_{F}^{s}=c_{s} / 3 \Lambda_{\chi}$ and $\left(\mu_{F}^{c}-\mu_{D}^{c}\right)=1 / 12 m_{c}$ we arrive at the first two terms of Eq.(15) in [7] in their notations and their Table I taken into account. Instead with

$$
\left(\mu_{F}^{c}+\frac{1}{3} \mu_{D}^{c}\right)=-\frac{1}{4} m_{c}, \quad\left(\mu_{F}-\frac{2}{3} \mu_{D}\right)=\left(\mu_{F}^{s}-\frac{2}{3} \mu_{D}^{s}\right)=\frac{c_{T}}{3 m_{c} \Lambda_{\chi}}
$$


one obtains the first two terms of Eq.(33) in [7] in their notations and their Table IV taken into account.

Results of the broken $S U(4)$ symmetry for the magnetic moments of the charm sextet as given by Eq.(9) can be matched to those of the quark model with four flavors as given in [1]. Really, upon using relations

$$
\begin{array}{r}
\mu_{F}=\frac{2}{3}\left(\mu_{u}-\mu_{d}\right), \quad \mu_{F}^{s}=\frac{2}{3}\left(\mu_{u}+2 \mu_{d}-3 \mu_{s}\right), \\
2\left(\mu_{F}^{c}-\mu_{D}^{c}\right)=\frac{4}{3} \mu_{u}+\frac{8}{3} \mu_{d}-\mu_{c},
\end{array}
$$

one obtains from Eq.(11) the relations of Eq.(6). It seems to be a nontrivial result as,e.g., there are no similar relations for charm antitriplet magnetic moments as well as for octet baryon ones.

\section{New formulae for the tree-level magnetic moments}

New formulae for the magnetic moments can be written in terms of the quark charges and $\mu_{F}$ 's and $\mu_{D}$ 's constants.

A magnetic moment of any hyperon with the $\Sigma\left(q q^{\prime}, q^{*}\right)$-like quark wave function containing two quarks $q, q^{\prime}$ in a biquark state and a single quark $q^{*}$ would have the form, in obvious notation:

$$
\mu_{\Sigma\left(q q^{\prime}, q^{*}\right)}=e_{q} \mu_{F}+e_{q^{\prime}} \mu_{F}^{\prime}+e_{q^{*}}\left(\mu_{F}^{*}-\mu_{D}^{*}\right)
$$

For the $\Sigma$-like baryons of the octet, that is for all of them but $\Lambda$ hyperon with $q, q^{\prime}, q^{*}=u, d, s$ and $\mu_{F}^{*}=\mu_{F}, \quad \mu_{D}^{*}=\mu_{D}$ one reproduces immediately Eq.(10). For the sextet charm baryons (which are all $\Sigma$-like ones) with $q, q^{\prime}=u, d, s, q^{*}=c$ and $\mu_{F}^{*}=\mu_{F}^{c}, \mu_{D}^{*}=\mu_{D}^{c}$, one reproduces the results given by Eq.(9) and quoted in Eq.(11).

One can write a formula for the magnetic moments of the $\Lambda$-like antitriplet charm hyperons containing two light quarks $q, q^{\prime}$ and one heavy quark $q^{*}$ as

$$
\mu_{\Lambda\left(q q^{\prime} q^{*}\right)}=e_{q}\left(\mu_{F}-\frac{2}{3} \mu_{D}\right)+e_{q^{\prime}}\left(\mu_{F}^{\prime}-\frac{2}{3} \mu_{D}^{\prime}\right)+e_{q^{*}}\left(\mu_{F}^{*}+\frac{1}{3} \mu_{D}^{*}\right) .
$$

With $q, q^{\prime}=u, d, s, q^{*}=c$ and $\mu_{F}^{*}=\mu_{F}^{c}, \mu_{D}^{*}=\mu_{D}^{c}$ one arrives at the result given by Eq.(9) and quoted in Eq.(13)

This formula is valid also for the octet $\Lambda$ magnetic moment. Taking $e_{q^{*}}=e_{s}=-\frac{1}{3}$ and $\mu_{F}=\mu_{F}^{*}, \mu_{D}=\mu_{D}^{*}$ one obtains the corresponding formula of the Eq.(10), namely, $\mu_{\Lambda}=-\mu_{D} / 3$.

\section{One-loop corrections to the charm baryon magnetic moments in $\mathrm{HHCPT}$}

An expression for the charm baryon magnetic moments in the HHCPT includes the tree-level formulae (with the corresponding counter terms) and the non-analytic corrections arising from the one-loop diagrams, involving $\pi$ and $K$ loops with 6 -plet $1 / 2,3^{*}$-plet $1 / 2$ and 6 -plet $3 / 2$ charm baryon insertions. One-loop corrections were evaluated in $[6,7]$ through effective Lagrangians 
involving directly sextet $1 / 2,3 / 2$ and $3^{*} 1 / 2$ charm baryon fields. But in order to be able to relate it to the case of the octet baryons it is more convenient for us to treat $1 / 2$ and $3 / 2$ spinors separately $[8,9,10]$. Also while considering couplings of the Goldstone bosons to baryons we use formally the $S U(4)$ symmetric Lagrangians Eqs. $(3,4,5)$ and then extract the part containing couplings of the charm baryons to the light Goldstone bosons. Thus magnetic moments of the charm baryons can be written in the form similar to those of the octet baryons $[8,9,10]$

$$
\mu_{B}=\mu_{B}^{0}+\frac{\pi}{\Lambda_{\chi}^{2}} \sum_{X=\pi, K}\left(\beta_{B}^{X}+\tilde{\beta}_{B}^{X}\right) m_{X} \equiv \mu_{B}^{0}+U(B),
$$

where $\mu_{B}^{0}$ are tree-level magnetic moments of the order $\mathcal{O}\left(1 / \Lambda_{\chi}\right)$ and/or $\mathcal{O}\left(1 / m_{c}\right)$, discussed in the previous section.

The $\beta_{B}^{\pi, K}$ and $\tilde{\beta}_{B}^{\pi, K}$ are the contributions from the pion and kaon loops with the photon line attached to the meson with intermediate baryon $1 / 2$ and $3 / 2$ states, respectively [8] . One-loop contributions into the charm baryon magnetic moments would be evaluated one by one and the corresponding Feynman diagrams would be presented similar to that done for the octet baryons [13].

\section{The 6-plet charm baryon magnetic moments}

1.1. Contribution into $\Sigma_{c}^{++}$magnetic moment

There are 6 diagrams, two of them with the 6(1/2)-plet baryons in the loop, two more diagrams with the $6(3 / 2)$-baryons in the loop and two last diagrams with the $3^{*}(1 / 2)$-plet baryons in the loop:
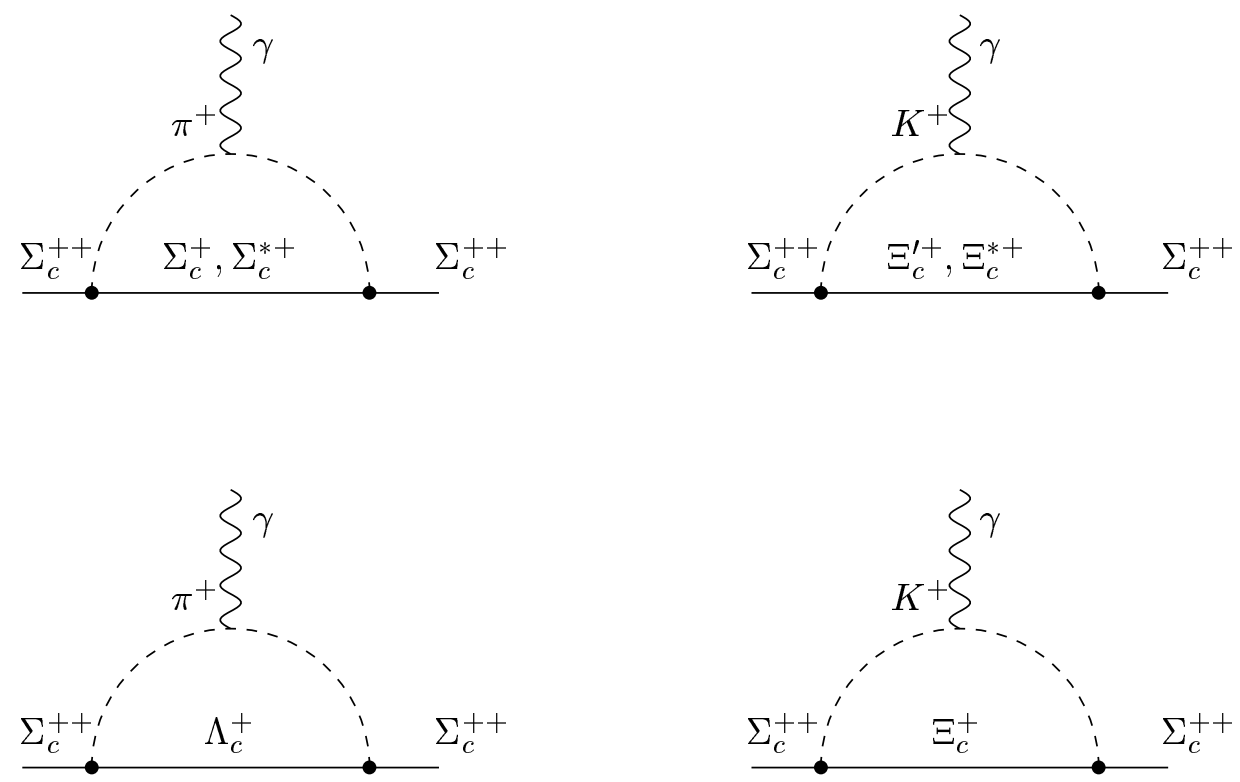

Fig.1.1

The coupling constants of the Goldstone bosons to the charm baryons in these diagrams are given by the Lagrangians (3) and (4) . The corresponding contribution upon taking account 
of the factors arriving from integration (see $[8,9,10]$ ) (that is a factor $(-1)$ in front of the $1 / 2$ contribution and a factor $1 / 3$ in front of the $3 / 2$ one) is written as ${ }^{3}$ :

$$
\begin{gathered}
\left.U\left(\Sigma_{c}^{++}\right)=\frac{\pi}{\Lambda_{\chi}^{2}} \sum_{X=\pi, K}\left(\beta_{\Sigma_{c}^{++}}^{X}+\tilde{\beta}_{\Sigma_{c}^{++}}^{X}\right) m_{X}\right)= \\
\frac{\pi}{\Lambda_{\chi}^{2}}\left[\left[-(\sqrt{2} F)^{2}+\frac{1}{3}\left(\frac{1}{6} \mathcal{C}^{2}\right)\right] m_{\pi}+\right. \\
\left.\left[-(\sqrt{2} F)^{2}+\frac{1}{3}\left(\frac{1}{6} \mathcal{C}^{2}\right)\right] m_{K}-\left(\sqrt{\frac{2}{3}} D\right)^{2} m_{\pi}-\left(\sqrt{\frac{2}{3}} D\right)^{2} m_{K}\right]= \\
\frac{\pi}{\Lambda_{\chi}^{2}}\left[-2 F^{2}-\frac{2}{3} D^{2}+\frac{2}{36} \mathcal{C}^{2}\right]\left(m_{\pi}+m_{K}\right) \Rightarrow-\frac{4}{3} \frac{\pi}{\Lambda_{\chi}^{2}}\left(m_{\pi}+m_{K}\right) D^{2}
\end{gathered}
$$

1.2. Contribution to $\Sigma_{c}^{+}$magnetic moment

There are 7 diagrams, three of them with the 6(1/2)-plet baryons in the loop, three more diagrams with the $6(3 / 2)$-baryons in the loop and the last one with the $3^{*}(1 / 2)$-plet baryons in the loop:
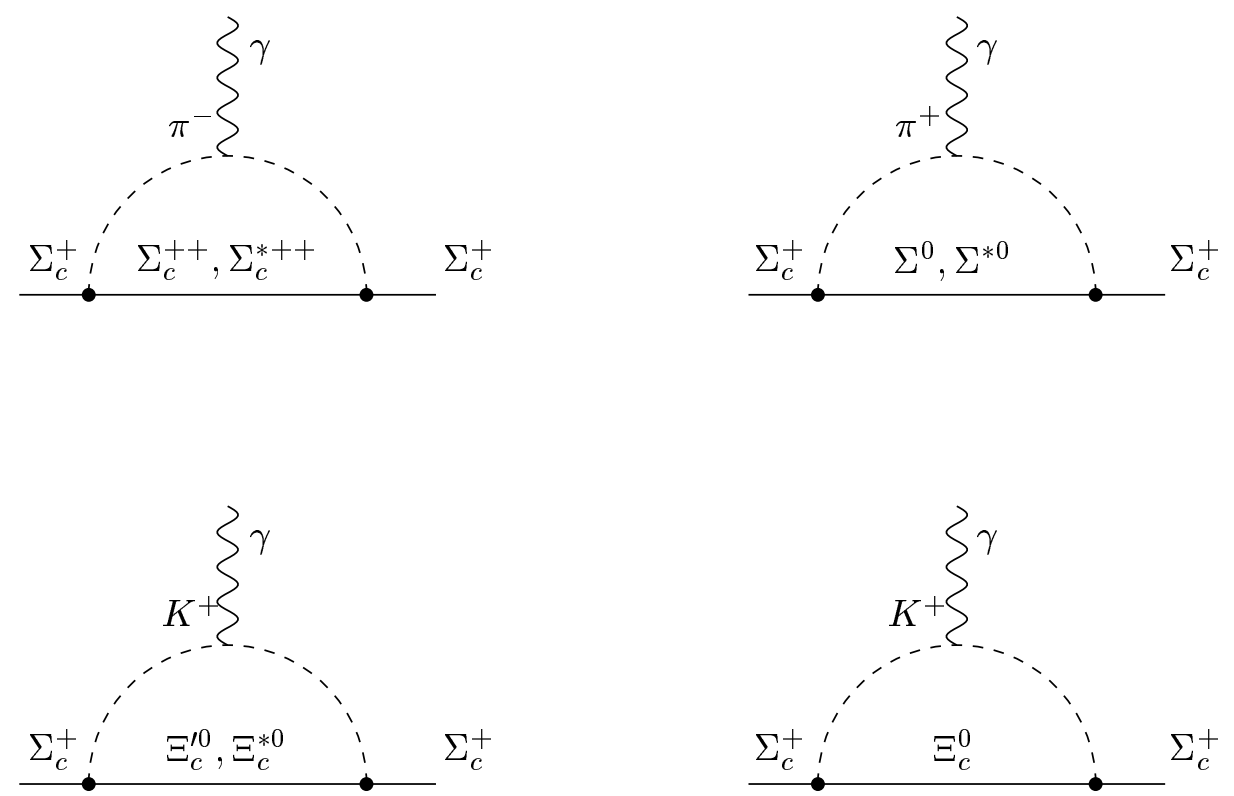

Fig.1.2

The contribution of these diagrams is calculated using the Lagrangians Eqs.(3) and (4):

$$
\begin{gathered}
U\left(\Sigma_{c}^{+}\right)=\frac{\pi}{\Lambda_{\chi}^{2}}\left[\left[(\sqrt{2} F)^{2}-\frac{1}{3}\left(\frac{1}{6} \mathcal{C}^{2}\right)\right] m_{\pi}+\left[-(\sqrt{2} F)^{2}+\frac{1}{3}\left(\frac{1}{6} \mathcal{C}^{2}\right)\right] m_{\pi}+\right. \\
\left.\left[-F^{2}+\frac{1}{3}\left(\frac{1}{12} \mathcal{C}^{2}\right)\right] m_{K}-\left(\sqrt{\frac{1}{3}} D\right)^{2} m_{K}\right]= \\
\frac{\pi}{\Lambda_{\chi}^{2}}\left[-F^{2}-\frac{1}{3} D^{2}+\frac{1}{36} \mathcal{C}^{2}\right] m_{K} \Rightarrow-\frac{2}{3} \frac{\pi}{\Lambda_{\chi}^{2}} m_{K} D^{2} .
\end{gathered}
$$

\footnotetext{
${ }^{3} \Rightarrow$ means throughout the paper that the $\mathrm{QM}$ limit $F=2 / 3 D, \quad \mathcal{C}=-2 D, \quad \mathcal{H}=-3 D$ is taken
} 
1.3. Contribution into $\Sigma_{c}^{0}$ magnetic moment

There are only 3 diagrams, with the 6(1/2)-plet baryon, the 6(3/2)-baryon in the loop and the last one with the $3^{*}(1 / 2)$-plet baryon in the $\pi$-loop:
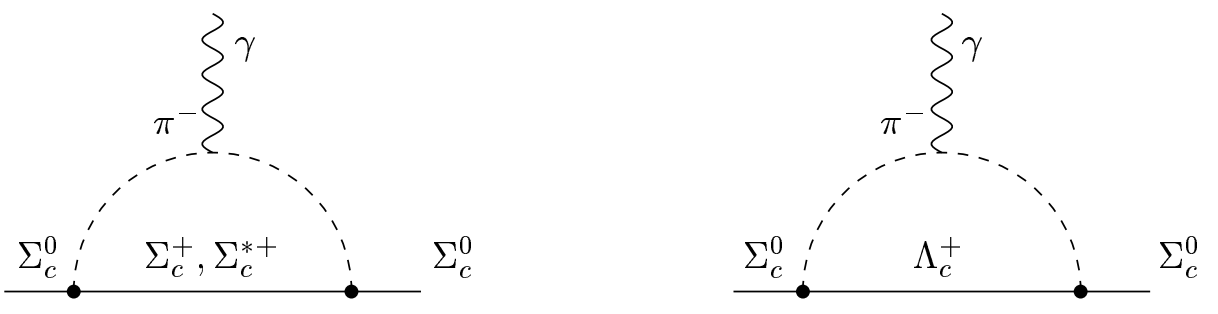

Fig.1.3

Upon using Eqs.(3) and (4):

$$
\begin{gathered}
U\left(\Sigma_{c}^{0}\right)=\frac{\pi}{\Lambda_{\chi}^{2}}\left[\left[(\sqrt{2} F)^{2}-\frac{1}{3}\left(\frac{1}{6} \mathcal{C}^{2}\right)+\left(-\sqrt{\frac{2}{3}} D\right)^{2}\right] m_{\pi}=\right. \\
\frac{\pi}{\Lambda_{\chi}^{2}}\left[2 F^{2}+\frac{2}{3} D^{2}-\frac{2}{36} \mathcal{C}^{2}\right] m_{\pi} \Rightarrow \frac{4}{3} \frac{\pi}{\Lambda_{\chi}^{2}} m_{\pi} D^{2} .
\end{gathered}
$$

1.4. Contribution into $\Xi_{c}^{\prime+}$ magnetic moment

There are 7 diagrams, three of them with the 6(1/2)-plet baryons in the loop, three more diagrams with the $6(3 / 2)$-baryons in the loop and the last one with the $3^{*}(1 / 2)$-plet baryons in the loop:
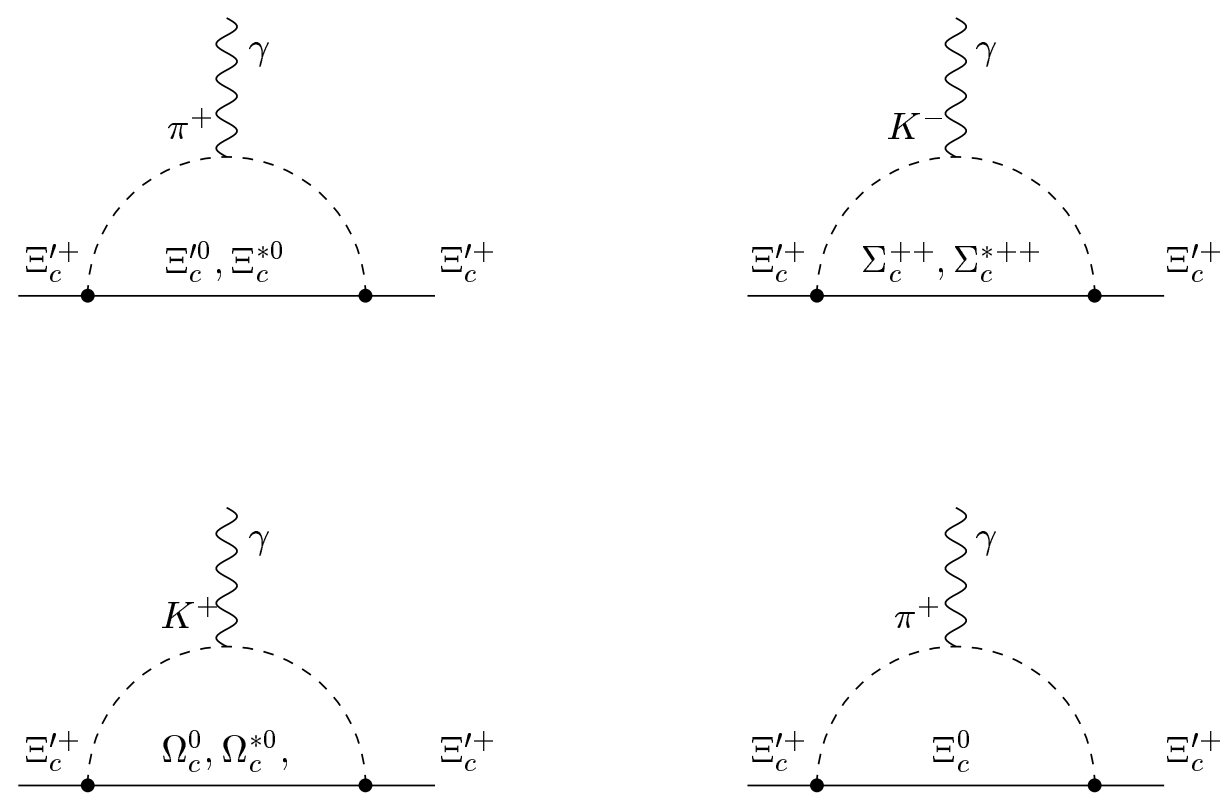

Fig.1.4

Upon using Eqs.(3) and (4): 


$$
\begin{aligned}
U\left(\Xi_{c}^{\prime+}\right)= & \frac{\pi}{\Lambda_{\chi}^{2}}\left[\left[-F^{2}+\frac{1}{3}\left(\frac{1}{6} \mathcal{C}^{2}\right)\right] m_{\pi}+\left[(\sqrt{2} F)^{2}-\frac{1}{3}\left(\frac{1}{12} \mathcal{C}^{2}\right)\right] m_{K}+\right. \\
& {\left.\left.[-\sqrt{2} F)^{2}+\frac{1}{3}\left(\frac{1}{12} \mathcal{C}^{2}\right)\right] m_{K}-\left(\sqrt{\frac{1}{3}} D\right)^{2} m_{\pi}\right]=} \\
& \frac{\pi}{\Lambda_{\chi}^{2}}\left[-F^{2}-\frac{1}{3} D^{2}+\frac{1}{36} \mathcal{C}^{2}\right] m_{\pi} \Rightarrow-\frac{2}{3} \frac{\pi}{\Lambda_{\chi}^{2}} m_{\pi} D^{2} .
\end{aligned}
$$

1.5. Contribution into $\Xi_{c}^{\prime 0}$ magnetic moment

Similar to the case of the $\Sigma_{c}^{++}$contribution there are 6 diagrams, two of them with the $6(1 / 2)$-plet baryons in the loop, two more diagrams with the 6(3/2)-baryons in the loop and two last diagrams with the $3^{*}(1 / 2)$-plet baryons in the loop:
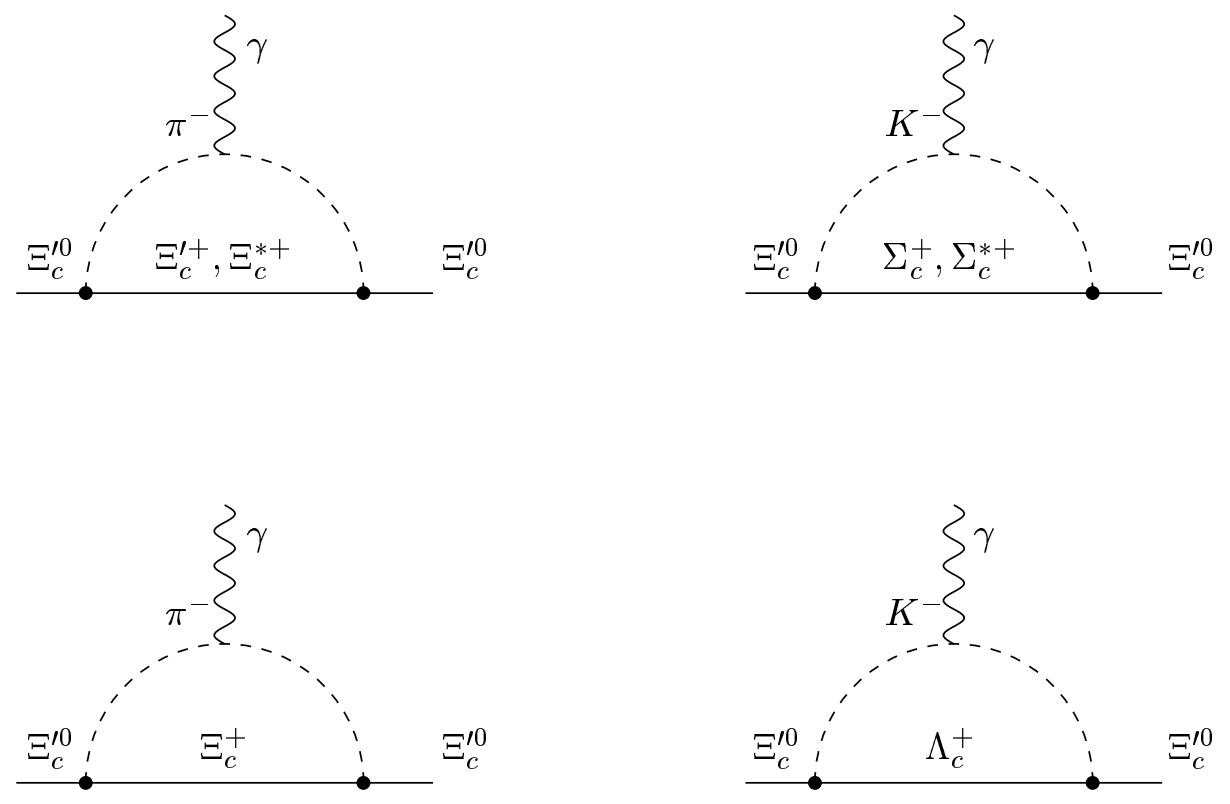

Fig.1.5

Upon using Eqs.(3) and (4):

$$
\begin{gathered}
U\left(\Xi_{c}^{\prime 0}\right)=\frac{\pi}{\Lambda_{\chi}^{2}}\left[\left[F^{2}-\frac{1}{3}\left(\frac{1}{12} \mathcal{C}^{2}\right)\right] m_{\pi}+\right. \\
\left.\left[F^{2}-\frac{1}{3}\left(\frac{1}{12} \mathcal{C}^{2}\right)\right] m_{K}+\left(\sqrt{\frac{1}{3}} D\right)^{2} m_{\pi}+\left(\sqrt{\frac{1}{3}} D\right)^{2} m_{K}\right]= \\
\frac{\pi}{\Lambda_{\chi}^{2}}\left[F^{2}+\frac{1}{3} D^{2}-\frac{1}{36} \mathcal{C}^{2}\right]\left(m_{\pi}+m_{K}\right) \Rightarrow \frac{2}{3} \frac{\pi}{\Lambda_{\chi}^{2}}\left(m_{\pi}+m_{K}\right) D^{2}
\end{gathered}
$$

1.6. Contribution into $\Omega_{c}^{0}$ magnetic moment

There are only 3 diagrams, with the 6(1/2)-plet baryon, the 6(3/2)-baryon and the last one with the $3^{*}(1 / 2)$-plet baryon in the $K$-loop: 

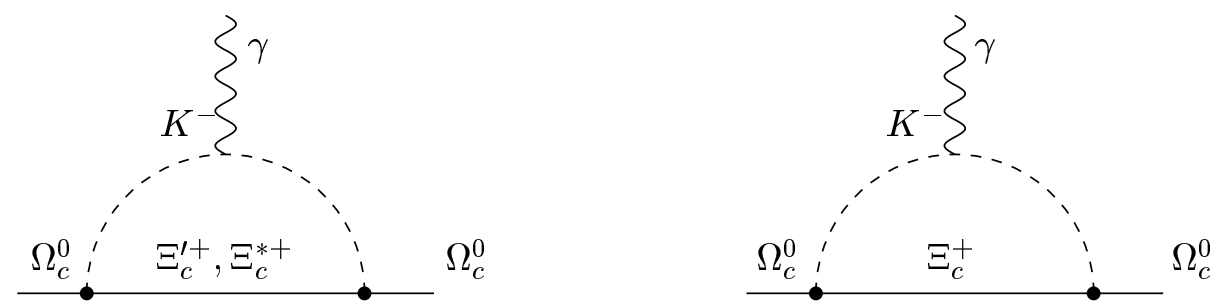

Fig.1.6

Upon using Eqs.(3) and (4):

$$
\begin{gathered}
U\left(\Omega_{c}^{0}\right)=\frac{\pi}{\Lambda_{\chi}^{2}}\left[\left[(\sqrt{2} F)^{2}-\frac{1}{3}\left(\frac{1}{6} \mathcal{C}^{2}\right)+\left(\sqrt{-\frac{2}{3}} D\right)^{2}\right] m_{K}=\right. \\
\frac{\pi}{\Lambda_{\chi}^{2}}\left[2 F^{2}+\frac{2}{3} D^{2}-\frac{2}{36} \mathcal{C}^{2}\right] m_{K} \Rightarrow \frac{4}{3} \frac{\pi}{\Lambda_{\chi}^{2}} m_{K} D^{2} .
\end{gathered}
$$

The $3^{*}$-plet charm baryon magnetic moments

2.1. Contribution into $\Lambda_{c}^{+}$magnetic moment

There are 7 diagrams, three of them with the 6(1/2)-plet baryons in the loop, three more diagrams with the $6(3 / 2)$-baryons in the loop and the last one with the $3^{*}(1 / 2)$-plet baryons in the loop:
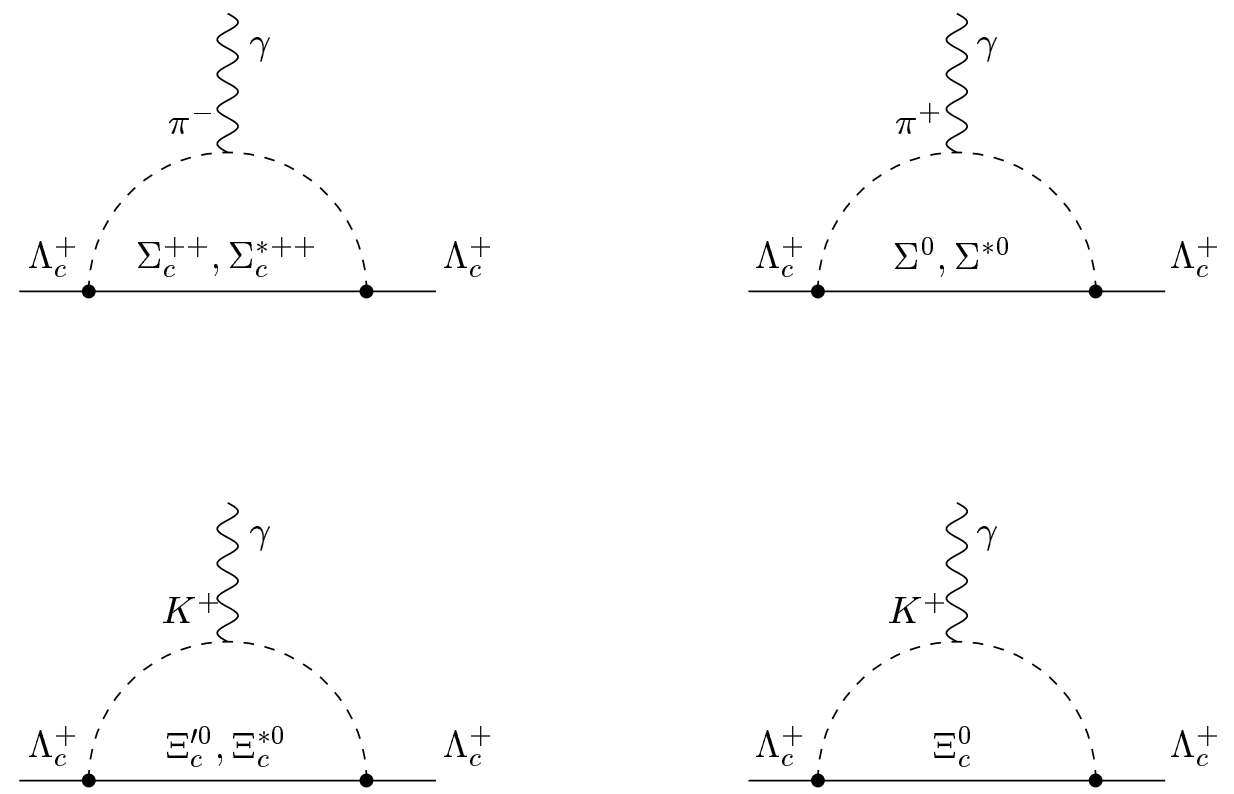

Fig.2.1

Upon using Eqs.(3) and (4):

$$
\begin{gathered}
U\left(\Lambda_{c}^{+}\right)=\frac{\pi}{\Lambda_{\chi}^{2}}\left[\left[\left(\sqrt{\frac{1}{3}} D\right)^{2}-\frac{1}{3}\left(\frac{1}{4} \mathcal{C}^{2}\right)\right] m_{\pi}+\left[-\left(\sqrt{\frac{1}{3}} D\right)^{2}+\frac{1}{3}\left(\frac{1}{4} \mathcal{C}^{2}\right)\right] m_{\pi}+\right. \\
\left.\left[-\left(\sqrt{\frac{1}{3}} D\right)^{2}+\frac{1}{3}\left(\frac{1}{4} \mathcal{C}^{2}\right)\right] m_{K}-\left(F-\frac{2}{3} D\right)^{2} m_{K}\right]=
\end{gathered}
$$




$$
\frac{\pi}{\Lambda_{\chi}^{2}}\left[-\frac{1}{3} D^{2}-\left(F-\frac{2}{3} D\right)^{2}+\frac{1}{12} \mathcal{C}^{2}\right] m_{K} \Rightarrow 0
$$

There is a non-zero contribution of the diagram with the $\Xi_{c}^{0}$-insertion proportional to the factor $\left(F-\frac{2}{3} D\right)^{2}$ which disappears in the quark model limit with $F=\frac{2}{3} D$. The remaining part of the one-loop contribution also disappears in this limit, but only because degenerated masses of the $3^{*} 1 / 2$ and sextet baryons are taken here. These diagrams give a non-zero contribution with nondegenerate masses $[6,7]$.

2.2. Contribution into $\Xi_{c}^{+}$magnetic moment

There are 7 diagrams, three of them with the 6(1/2)-plet baryons in the loop, three more diagrams with the $6(3 / 2)$-baryons in the loop and the last one with the $3^{*}(1 / 2)$-plet baryons in the loop:
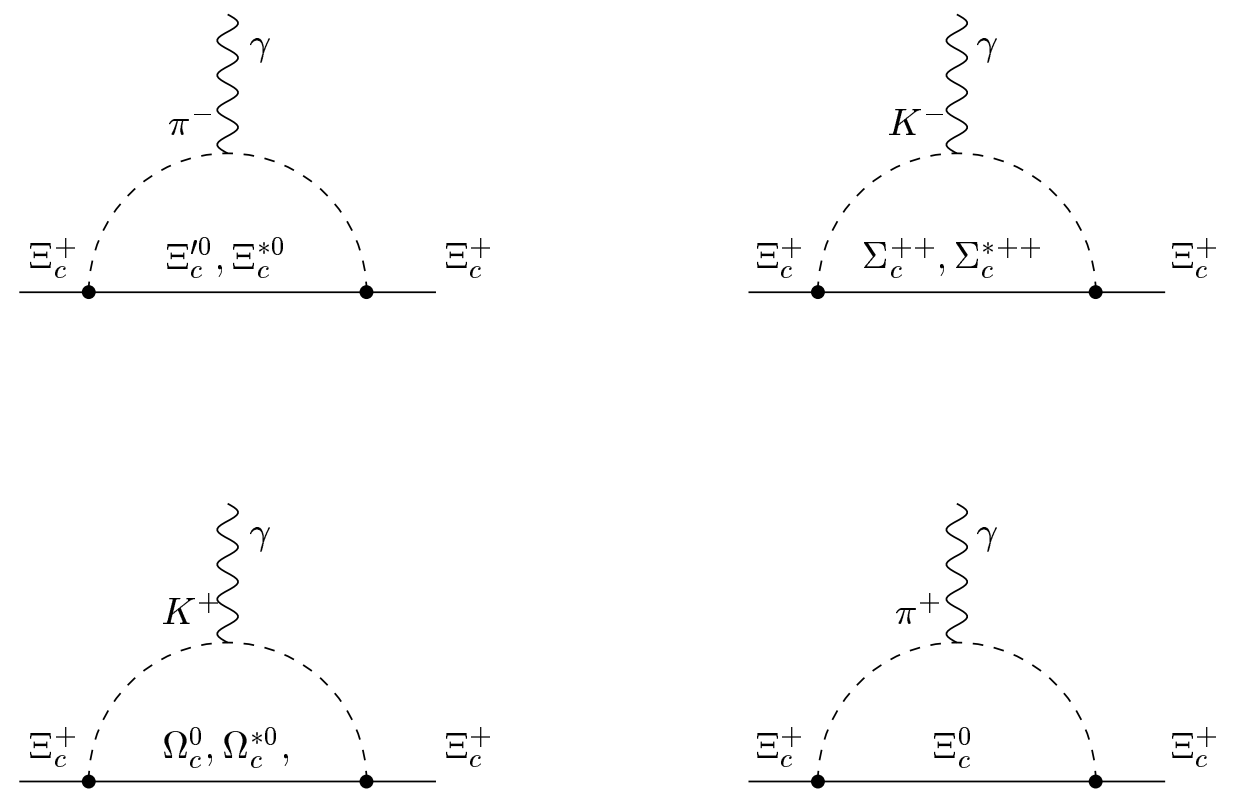

Fig. 2.2

Upon using Eqs.(3) and (4):

$$
\begin{gathered}
U\left(\Xi_{c}^{+}\right)=\frac{\pi}{\Lambda_{\chi}^{2}}\left[\left[-\left(\sqrt{\frac{1}{3}} D\right)^{2}+\frac{1}{3}\left(\frac{1}{4} \mathcal{C}^{2}\right)\right] m_{\pi}+\left[\left(\sqrt{\frac{1}{3}} D\right)^{2}-\frac{1}{3}\left(\frac{1}{4} \mathcal{C}^{2}\right)\right] m_{K}+\right. \\
\left.\left[-\left(\sqrt{\frac{1}{3}} D\right)^{2}+\frac{1}{3}\left(\frac{1}{4} \mathcal{C}^{2}\right)\right] m_{K}-\left(F-\frac{2}{3} D\right)^{2} m_{\pi}\right]= \\
\frac{\pi}{\Lambda_{\chi}^{2}}\left[-\frac{1}{3} D^{2}-\left(F-\frac{2}{3} D\right)^{2}+\frac{1}{12} \mathcal{C}^{2}\right] m_{\pi} \Rightarrow 0
\end{gathered}
$$

There is also a non-zero contribution of the diagram with the $\Xi_{c}^{0}$-insertion proportional to the factor $\left(F-\frac{2}{3} D\right)^{2}$ which disappears in the quark model limit with $F=\frac{2}{3} D$. Also here the remaining part of the one-loop contribution disappears in this limit.

2.3. Contribution into $\Xi_{c}^{0}$ magnetic moment 
Similar to the cases of the $\Sigma_{c}^{++}$and $\Xi_{c}^{\prime 0}$ contributions there are 6 diagrams, two of them with the 6(1/2)-plet baryons in the loop, two more diagrams with the 6(3/2)-baryons in the loop and two last diagrams with the $3^{*}(1 / 2)$-plet baryons in the loop:
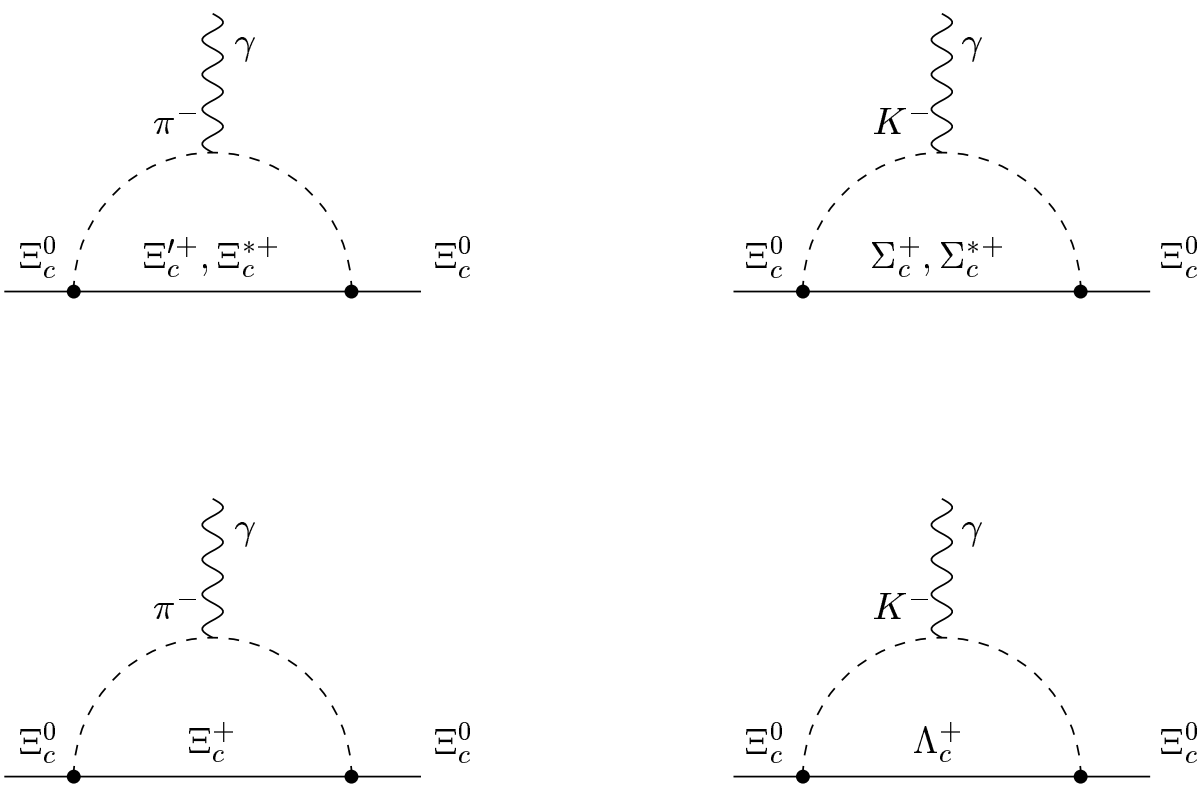

Fig. 2.3

Upon using Eqs.(3) and (4):

$$
\begin{gathered}
U\left(\Xi_{c}^{0}\right)=\frac{\pi}{\Lambda_{\chi}^{2}}\left[\left[\left(\sqrt{\frac{1}{3}} D\right)^{2}-\frac{1}{3}\left(\frac{1}{4} \mathcal{C}^{2}\right)\right] m_{\pi}+\right. \\
\left.\left.\left[\sqrt{\frac{1}{3}} D\right)^{2}-\frac{1}{3}\left(\frac{1}{4} \mathcal{C}^{2}\right)\right] m_{K}+\left(F-\frac{2}{3} D\right)^{2} m_{\pi}+\left(F-\frac{1}{3} D\right)^{2} m_{K}\right]= \\
\frac{\pi}{\Lambda_{\chi}^{2}}\left[\frac{1}{3} D^{2}+\left(F-\frac{2}{3} D\right)^{2}-\frac{1}{12} \mathcal{C}^{2}\right]\left(m_{\pi}+m_{K}\right) \Rightarrow 0
\end{gathered}
$$

There is a non-zero contribution of the diagram with the $\Lambda_{c}^{+}$-insertion proportional to the factor $\left(F-\frac{2}{3} D\right)^{2}$ which disappears in the quark model limit with $F=\frac{2}{3} D$. Also here the remaining part of the one-loop contribution disappears in this limit.

\section{$5 \quad$ Results and discussion}

Finally one-loop contributions lead to the following expressions for the magnetic moments of the sextet $1 / 2$ :

$$
\begin{gathered}
\mu_{\Sigma_{c}^{++}}=\mu_{\Sigma_{c}^{++}}^{0}+2 \kappa\left(m_{\pi}+m_{K}\right), \\
\mu_{\Sigma_{c}^{+}}=\mu_{\Sigma_{c}^{+}}^{0}+\kappa m_{K}, \quad \mu_{\Sigma_{c}^{0}}=\mu_{\Sigma_{c}^{0}}^{0}-2 \kappa m_{\pi}, \\
\mu_{\Xi_{c}^{\prime+}}=\mu_{\Xi_{c}^{\prime+}}^{0}+\kappa m_{\pi}, \quad \mu_{\Xi_{c}^{\prime 0}}=\mu_{\Xi_{c}^{\prime 0}}^{0}-\kappa\left(m_{\pi}+m_{K}\right), \\
\mu_{\Omega_{c}^{0}}=\mu_{\Omega_{c}^{0}}^{0}-2 \kappa m_{K},
\end{gathered}
$$


where

$$
\kappa=\frac{\pi}{\Lambda_{\chi}^{2}}\left[-F^{2}-\frac{1}{3} D^{2}+\frac{1}{36} \mathcal{C}^{2}\right] \rightarrow-\frac{2}{3} \frac{\pi}{\Lambda_{\chi}^{2}}
$$

One-loop contributions considered in [7] and in this work do not change the relations of Eq.(8):

$$
\begin{gathered}
\mu_{\Sigma_{c}^{++}}+\mu_{\Sigma_{c}^{0}}=2 \mu_{\Sigma_{c}^{+}}, \\
\mu_{\Sigma_{c}^{++}}+\mu_{\Omega_{c}^{0}}=2 \mu_{\Xi_{c}^{\prime+}}, \\
\mu_{\Sigma_{c}^{++}}+2 \mu_{\Xi_{c}^{\prime 0}}=\mu_{\Sigma_{c}^{0}}+2 \mu_{\Xi_{c}^{\prime+}} .
\end{gathered}
$$

The formulae for the magnetic moments of the sextet charm baryons in the quark model (see Eq.(6)) remain essentially the same with the account of the one-loop corrections considered if one renormalizes effectively the light quark magnetic moments in the following way:

$$
\begin{gathered}
\mu_{u} \rightarrow \mu_{u}-\frac{3}{2} \kappa\left(m_{\pi}+m_{K}\right), \\
\mu_{d} \rightarrow \mu_{d}+\frac{3}{2} \kappa m_{\pi}, \quad \mu_{s} \rightarrow \mu_{s}+\frac{3}{2} \kappa m_{K} .
\end{gathered}
$$

An analogous conclusion is valid for the magnetic moments of the charm baryons obtained in [7]. One can proceed in a similar way in the unitary symmetry model, renormalizing $\mu_{D, F}$ 's upon using Eq.(14). One-loop contributions yield the following expressions for the magnetic moments of antitriplet $1 / 2$ :

$$
\begin{array}{r}
\mu_{\Lambda_{c}^{+}}=\mu_{\Lambda_{c}^{+}}^{0}+\alpha m_{K}, \\
\mu_{\Xi_{c}^{+}}=\mu_{\Xi_{c}^{+}}^{0}+\alpha m_{\pi}, \quad \mu_{\Xi_{c}^{0}}=\mu_{\Xi_{c}^{0}}^{0}-\alpha\left(m_{\pi}+m_{K}\right),
\end{array}
$$

where

$$
\alpha=-\frac{\pi}{\Lambda_{\chi}^{2}}\left[\frac{1}{3} D^{2}+\left(F-\frac{2}{3} D\right)^{2}-\frac{1}{12} \mathcal{C}^{2}\right]
$$

The one-loop contributions given by the diagrams of the Figs.2.1-3 disappear in the quark model limit $\left(F=\frac{2}{3} D, \mathcal{C}=-2 D\right)$. But in the case of a large unitary symmetry breaking, naturally expected for models with more than 3 flavors, they would give non-zero contribution and break the NRQM prediction of [1] (see Eq.(8)) at the level inferior to that considered in $[6,7]$.

Instead in the unitary symmetry model the one-loop corrections can be hidden into the constants $\mu_{D, F}, \mu_{D, F}^{s, c}$ of Eq.(13) by a renormalization procedure

$$
\begin{aligned}
& \left(\mu_{F}-\frac{2}{3} \mu_{D}\right) \rightarrow\left(\mu_{F}-\frac{2}{3} \mu_{D}\right)-\alpha\left(2 m_{\pi}+m_{K}\right), \\
& \left(\mu_{F}^{s}-\frac{2}{3} \mu_{D}^{s}\right) \rightarrow\left(\mu_{F}^{s}-\frac{2}{3} \mu_{D}^{s}\right)+\alpha\left(m_{\pi}-4 m_{K}\right) \\
& \left(\mu_{F}^{c}+\frac{1}{3} \mu_{D}^{c}\right) \rightarrow\left(\mu_{F}^{c}+\frac{1}{3} \mu_{D}^{c}\right)+\alpha\left(m_{\pi}-m_{K}\right) .
\end{aligned}
$$

Really, applying it to Eq.(16) one just rederives Eq.(13). 
Thus for the magnetic moments of the sextet $1 / 2$ baryons with the one-loop corrections the old-fashion NRQM picture or that of the broken unitary symmetry model effectively emerges.

The one-to-one correspondence between HHCPT results and those of NRQM proved here is a rather unexpected result. For example, one-loop contributions to the magnetic moments of the octet baryons $[8,9,10]$ do not reveal structure either of the NRQM or of the unitary symmetry model. So while analysing the magnetic moments of the sextet $1 / 2$ charm baryons it would be difficult to distinguish between the tree-level contributions and those of the one-loop corrections.

As for the magnetic moments of the $3^{*} 1 / 2$ charm baryons an eventual deviation from the quark model prediction [1] could be connected either with the unitary symmetry breaking terms or with the high-order corrections, as those considered in [6] or [7].

Only a more general picture which embraces octet and eventually decuplet baryon magnetic moments would be able to answer the question on relative importance of the one-loop corrections to the magnetic moments of the charm baryons in the framework of the chiral perturbation theory.

\section{Acknowledgments}

I am grateful to V.M.Dubovik, F.Hussain and G.Thompson for discussions. I thank J.Bernabeu for the e-mail discussion.

I would like to thank Professor S.Randjbar-Daemi and the High Energy Section of the Abdus Salam ICTP (Trieste, Italy) where the main part of this work was done for the hospitality extended to me.

The financial support of the Abdus Salam International Centre for Theoretical Physics (Trieste, Italy) is gratefully acknowledged. 


\section{References}

[1] R.J. Johnson and M.Shah-Jahan Phys.Rev. D15, 1400 (1977)

[2] A.I. Choudhury, V. Joshi, Phys.Rev. D13, 3115 (1976); ibid., 3120.

[3] N. Isgur and M.B. Wise, Phys. Lett. B232, 113 (1989); Nucl.Phys. B348, 276 (1991).

[4] H. Georgi, Phys. Lett. B240, 447 (1990); Nucl.Phys. B348, 293 (1991).

[5] F. Hussain, J.G. Körner, G. Thompson, Ann. Phys. 206, 334 (1991).

[6] M.J. Savage, Phys. Lett. 326, 303 1994).

[7] M.C. Banuls, I. Scimemi,J. Bernabeu, V. Gimenez,and A. Pich, Phys. Rev. D61, 074007 (2000).

[8] E. Jenkins, M. Luke, A. Manohar and M.J. Savage, Phys. Lett. B302, 482 (1993); ibid., B388, 866 (1996)(E).

[9] P. Ha and L. Durand, Phys. Rev. D58, 093008 (1998); L. Durand and P. Ha, Phys. Rev. D58, 013010 (1998); P. Ha, Phys. Rev. D58, 113003 (1998).

[10] S.J. Puglia and M.J. Ramsey-Musolf, Phys. Rev. D62, 034010 (2000).

[11] S. Coleman, S.L. Glashow, Phys. Rev. Lett. 6, 423 (1961).

[12] E.N. Bukina, V.M. Dubovik, V.S. Zamiralov, Vestnik. Mosk. Universiteta. 2, 3 (2000); E.N. Bukina, J. Mod. Phys.A, 14, 2525 (1999).

[13] V.S. Zamiralov, NPI MSU Preprint 2001-8/648, Nuclear Physics Institute, Moscow State University, 119899 Moscow, Russia. 